\title{
Interval-Valued Linear Model
}

\author{
Xun Wang ${ }^{1,2}$, Shoumei $\mathrm{Li}^{1, *}$, Thierry Denoux ${ }^{2}$ \\ ${ }^{1}$ Department of Applied Mathematics, \\ Beijing University of Technology, \\ Beijing, 100124, China \\ ${ }^{2}$ Heudiasyc, UMR CNRS 7253, \\ Université de Technologie de Compiègne, CNRS, \\ Compiègne, 60200, France
}

Received 30 May 2014

Accepted 15 July 2014

\begin{abstract}
This paper introduces a new type of statistical model: the interval-valued linear model, which describes the linear relationship between an interval-valued output random variable and real-valued input variables. Firstly, notions of variance and covariance of set-valued and interval-valued random variables are introduced. Then, we give the definition of the interval-valued linear model and its least square estimator (LSE), as well as some properties of the LSE. Thirdly, we show that, whereas the best linear unbiased estimation does not exist, the best binary linear unbiased estimator exists and it is the LSE. Finally, we present simulation experiments and an application example regarding temperatures of cities affected by their latitude, which illustrates the application of the proposed model.
\end{abstract}

Keywords: interval-valued linear model, least square estimation, best binary linear unbiased estimation, $D_{p}$ metric.

\section{Introduction}

Traditional statistical models have played a significant role in a wide range of areas. However, in real life situations, many problems cannot be handled by traditional statistical models due to imperfectness of data. Therefore, specialized statistical techniques are needed. In many practical cases, we have to face a particular kind of imperfect data: interval-valued data ${ }^{8,9,13}$.

Interval-valued data may represent uncertainty or variability. In the former case, the interval data represent incomplete observations, i.e., we just know the true data belong to a range (an interval), rather than precise values. For example, researchers test the service life of a group of products, such as light bulbs. Since testing time is very long, they cannot stay in the laboratory at any time. Alternatively, they could come to the laboratory to observe how many bulbs are burnt out every two or three hours. Thus, the data of service life of bulbs are interval-valued. In contrast, in the variability case, an interval is not interpreted as a set containing a single true value, but the observation themselves are interval-valued. For instance, a weather forecast typically provides the highest and lowest temperature of the next day, which is an interval including almost all the useful information about tomorrow's temperature. This interval reflects the variability of temperature in one

*Corresponding author, email: lisma@bjut.edu.cn. The research is supported by NSFC (No. 11171010). 
day.

The linear model is probably the simplest and most frequently-used statistical model. It describes a random output variable influenced by a few input variables and an error term in a linear way. In this paper, we consider the situation of interval-valued observations, i.e., the output variable is an intervalvalued random variable, which is determined by real-valued variables in a linear way. This intervalvalued linear model could play a significant role in dealing with imperfect data, e.g., to investigate how (interval-valued) temperature is impacted by (pointvalued) intensity of solar radiation, air pressure, latitude of location, or the statistical relationship between interval-valued service life of light bulbs and point-valued properties of materials used in making bulbs.

Interval-valued random variables are a special kind of set-valued random variables, whose values are compact convex subsets of real line $\mathbb{R}^{1}$. Since we have at our disposal many results on the theory of set-valued random variables ${ }^{18,19,29}$, this is a suitable framework to tackle the problem addressed in this paper. Until recently, however, there has been only a few works discussing the variance and covariance of set-valued random variables, since the difference between two sets is difficult to define and the hyperspace (e.g., the space of all intervals) is not linear with respect to addition and multiplication. $\mathrm{Vital}^{23}$ studied the metric for compact convex sets via the support functions. In 2005, Yang and $\mathrm{Li}^{27}$, Yang ${ }^{28}$ investigated the $d_{p}$ metric for sets and the $D_{p}$ metric in the space of set-valued random variables. They proposed to use the $D_{p}$ metric to define the variance and covariance of set-valued and interval-valued random variables, which proved to be a good approach to deal with this problem. In Chapter 5 of Yang ${ }^{28}$, the author also built a linear regression model with interval-valued regression coefficients. The underlying space in the above two papers is $\mathbb{R}^{d}$. In 2008 , Blanco et al. ${ }^{4}$ defined $d_{K^{-}}$ variance for interval-valued random variables with underlying space being $\mathbb{R}^{1}$, which is a special case of Yang and $\mathrm{Li}^{27}$ and Yang ${ }^{28}$.

Other authors studied interval-valued and setvalued statistical models. Tanaka and Lee ${ }^{21}$ intro- duced the interval linear regression model, which is not based on the interval-valued random variable framework, and estimated the coefficients using a quadratic optimization method. Blanco-Fernandez et al. ${ }^{5}$ and Sinova et al. ${ }^{20}$ investigated the linear relationship between two interval-valued random variables considering the input variable as two realvalued random variables (center and radius of the interval). They gave the LSE of the coefficients under the $d_{2}$ metric of intervals. Blanco-Fernandez et al. ${ }^{6}$ studied the strong consistency and asymptotic distributions of the LSE. Hsu and $\mathrm{Wu}^{14}$ investigated interval-valued time series and gave three evaluation criteria of estimation and forecast efficiency for interval-valued time series. Wang and $\mathrm{Li}^{24}$ introduced a new type of interval-valued time series (the interval autoregressive time series model) and gave the estimation method of parameters and forecast method based on the evaluation criteria. Wang and $\mathrm{Li}^{25}$ investigated set-valued and interval-valued stationary time series, which is based on the definition of variance and covariance of set-valued and interval-valued random variables introduced in Yang and $\mathrm{Li}^{27}$ and Yang ${ }^{28}$.

In this paper, we start with the set-valued framework and consider interval-valued random variables as a special case. We then introduce the intervalvalued linear model and its LSE, prove its unbiasedness and discuss the best binary unbiased estimation. Treating an interval-valued random variable as two separate point-valued random variables (the left- and right-endpoints of the interval, or the center and radius of the interval) has some drawbacks. One reason is that it is possible to obtain estimation or forecast results such that the left-endpoint is larger than the right-endpoint, because these two linear models are unrelated. In this paper, we also show the limitation of using two separate linear models in terms of forecast efficiency via a simulation experiment.

This paper is a complete version of the results presented by the authors ${ }^{26}$. The organization of this paper is as follows. In Section 2, we define the variance and covariance of set-valued random variables based on the $d_{p}$ metric for sets and the $D_{p}$ metric for interval-valued random variables. In Section 3, we introduce the interval-valued linear model and its 
LSE, prove the unbiasedness of this LSE and give the covariance matrix of this estimator. Section 4 shows that the best linear unbiased estimation does not exist in general, but the best binary linear unbiased estimation (BBLUE) exists, is unique and equal to the LSE. In Section 5, we present a simulation study to show the methodology, and illustrate the efficiency of estimation method introduced in Sections 3 and 4 . We then present another simulation experiment to compare our model with using two separate linear models. Finally, in Section 6, we use the interval-valued linear model to investigate the relationship between city temperature and latitude. This example also shows how this model can be used to deal with some practical problems.

\section{Variance and Covariance of Set-Valued Random Variables}

\section{1. $d_{p}$ Metric of Sets}

In this section, we assume that $(\Omega, \mathscr{A}, P)$ is a probability space, $(\mathscr{X},\|\cdot\| \mathscr{X})$ is a Banach space, $\mathbf{K}(\mathscr{X})$ is the family of all nonempty closed subsets of $\mathscr{X}$, $\mathbf{K}_{k c}(\mathscr{X})$ is the family of all nonempty compact convex subsets of $\mathscr{X}$.

For any $A, B \in \mathbf{K}(\mathscr{X}), \lambda \in \mathbb{R}$, define

$$
\begin{gathered}
A+B=\{a+b: a \in A, b \in B\}, \\
\lambda A=\{\lambda a: a \in A\},
\end{gathered}
$$

and denote

$$
A \oplus B=\operatorname{cl}\{a+b: a \in A, b \in B\} .
$$

If $A, B \in \mathbf{K}_{k c}(\mathscr{X})$, then $A+B \in \mathbf{K}_{k c}(\mathscr{X})$.

For each $A \in \mathbf{K}_{k c}(\mathscr{X})$, the support function is defined by

$$
s\left(x^{*}, A\right)=\sup \left\{x^{*}(a): a \in A\right\}, \quad x^{*} \in \mathscr{X}^{*},
$$

where $\mathscr{X}^{*}$ is the dual space of $\mathscr{X}$, i.e., the set of all bounded linear functionals on $\mathscr{X}$. For example, if $\mathscr{X}=\mathbb{R}^{1}, \mathscr{X}^{*}=\mathbb{R}^{1}$. Take an interval $[a, b]$ with $0 \leqslant a<b, x \in \mathbb{R}^{1}$, then the support function is $s(x,[a, b])=\left\{\begin{array}{l}b x, x \geqslant 0 \\ a x, x<0\end{array}\right.$. The support function has the following properties:

$$
s\left(x^{*}, A \oplus B\right)=s\left(x^{*}, A+B\right)=s\left(x^{*}, A\right)+s\left(x^{*}, B\right),
$$

$$
s\left(x^{*}, \lambda A\right)=\lambda s\left(x^{*}, A\right), \lambda \geqslant 0 .
$$

For $1 \leqslant p<\infty$, take $A, B \in \mathbf{K}_{k c}(\mathscr{X})$. We define the metric $d_{p}$ on $\mathbf{K}_{k c}(\mathscr{X})^{2,18,27}$ by

$$
d_{p}(A, B)=\left[\int_{S^{*}}\left|s\left(x^{*}, A\right)-s\left(x^{*}, B\right)\right|^{p} d \mu\right]^{1 / p},
$$

where $S^{*}$ is the unit sphere of $\mathscr{X}^{*}$, i.e. $S^{*}=$ $\left\{x^{*} \in \mathscr{X}^{*}:\left\|x^{*}\right\|_{\mathscr{X}^{*}}=1\right\}, \mu$ is a measure on $\left(\mathscr{X}^{*}, \mathscr{B}\left(\mathscr{X}^{*}\right)\right)$.

Remark 2.1. If $\mathscr{X}=\mathbb{R}^{1}$, then $\mathbf{K}_{k c}\left(\mathbb{R}^{1}\right)=\{[a, b]$ : $-\infty<a \leqslant b<\infty\}$ is the family of all intervals on $\mathbb{R}^{1}$. If $A_{1}, A_{2} \in \mathbf{K}_{k c}\left(\mathbb{R}^{1}\right)$ with $A_{1}=\left[a_{1}, b_{1}\right]=\left(c_{1} ; r_{1}\right)$, $A_{2}=\left[a_{2}, b_{2}\right]=\left(c_{2} ; r_{2}\right)$, where $c_{i}=\left(a_{i}+b_{i}\right) / 2$ and $r_{i}=\left(b_{i}-a_{i}\right) / 2$ for $i=1,2$, then

$$
\begin{gathered}
A_{1}+A_{2}=\left[a_{1}+a_{2}, b_{1}+b_{2}\right]=\left(c_{1}+c_{2} ; r_{1}+r_{2}\right), \\
k A_{1}=\left(k c_{1} ;|k| r_{1}\right),
\end{gathered}
$$

and

$$
\begin{aligned}
& d_{p}\left(A_{1}, A_{2}\right)=\left[\left|a_{2}-a_{1}\right|^{p}+\left|b_{2}-b_{1}\right|^{p}\right]^{1 / p} \\
= & {\left[\left|\left(c_{2}-c_{1}\right)-\left(r_{2}-r_{1}\right)\right|^{p}+\left|\left(c_{2}-c_{1}\right)+\left(r_{2}-r_{1}\right)\right|^{p}\right]^{\frac{1}{p}} . }
\end{aligned}
$$

Theorem 2.1. ${ }^{27}\left(\boldsymbol{K}_{k c}\left(\mathbb{R}^{d}\right), d_{p}\right)$ is a complete, separable metric space for each $p \in[1, \infty)$.

\section{2. $D_{p}$ Metric Space of Set-Valued Random Variables}

A set-valued mapping $F: \Omega \rightarrow \mathbf{K}(\mathscr{X})$ is called a setvalued random variable ${ }^{11,18}$ if, for each open subset $O$ of $\mathscr{X}, F^{-1}(O) \in \mathscr{A}$, where $F^{-1}(O)=\{\omega \in \Omega$ : $F(\omega) \cap O \neq \emptyset\}$ and $\emptyset$ is the empty set. Any two setvalued random variables are considered identical if $F_{1}(\omega)=F_{2}(\omega)$ for almost every $\omega \in \Omega$ (for short, denoted by "a.s. $(P)$ ").

Let $\mathscr{U}\left[\Omega, \mathbf{K}_{k c}(\mathscr{X})\right]$ denote the family of setvalued random variables taking values in $\mathbf{K}_{k c}(\mathscr{X})$. The $D_{p}$ metric $^{27}$ with respect to set-valued random variables is defined by

$$
D_{p}\left(F_{1}, F_{2}\right)=\left[E\left(d_{p}^{p}\left(F_{1}(\omega), F_{2}(\omega)\right)\right)\right]^{1 / p},
$$

where $F_{1}, F_{2} \in \mathscr{U}\left[\Omega, \mathbf{K}_{k}(\mathscr{X})\right]$. 
Remark 2.2. If $\mathscr{X}=\mathbb{R}^{1}, \quad \mathscr{U}\left[\Omega, \mathbf{K}_{k c}(\mathscr{X})\right]=$ $\mathscr{U}\left[\Omega, \mathbf{K}_{k c}\left(\mathbb{R}^{1}\right)\right]$ is the family of all interval-valued random variables. For $F_{i} \in \mathscr{U}\left[\Omega, \mathbf{K}_{k c}\left(\mathbb{R}^{1}\right)\right]$, $F_{i}(\omega)=\left[f_{i}(\omega), g_{i}(\omega)\right]=\left(c_{i}(\omega) ; r_{i}(\omega)\right)$, where $f_{i}(\omega), g_{i}(\omega)$ are random variables and $f_{i}(\omega) \leqslant$ $g_{i}(\omega)$, and $c_{i}(\omega)=\left(f_{i}(\omega)+g_{i}(\omega)\right) / 2, r_{i}(\omega)=$ $\left(g_{i}(\omega)-f_{i}(\omega)\right) / 2, i=1,2$. By the definition of $D_{p}$, we have

$$
\begin{aligned}
& D_{p}\left(F_{1}(\omega), F_{2}(\omega)\right) \\
= & {\left[E\left|f_{2}(\omega)-f_{1}(\omega)\right|^{p}+E\left|g_{2}(\omega)-g_{1}(\omega)\right|^{p}\right]^{1 / p} } \\
= & {\left[E\left|\left(c_{2}(\omega)-c_{1}(\omega)\right)-\left(r_{2}(\omega)-r_{1}(\omega)\right)\right|^{p}\right.} \\
& \left.+E\left|\left(c_{2}(\omega)-c_{1}(\omega)\right)+\left(r_{2}(\omega)-r_{1}(\omega)\right)\right|^{p}\right]^{1 / p} .
\end{aligned}
$$

Denote $\quad \mathscr{L}^{p}\left[\Omega, \mathbf{K}_{k c}(\mathscr{X})\right]=\{F: F \in$ $\left.\mathscr{U}\left[\Omega, \mathbf{K}_{k c}(\mathscr{X})\right], E\left[\|F\|_{d_{p}}^{p}\right]<+\infty\right\}$. Then we have the following theorem:

Theorem 2.2. ${ }^{27}\left(\mathscr{L}^{p}\left[\Omega, K_{k c}\left(\mathbb{R}^{d}\right)\right], D_{p}\right)$ is a complete metric space for each $p \in[1, \infty)$.

\subsection{Variance and Covariance of Set-Valued Random Variables}

The expectation of a set-valued random variable $F$ was introduced by Aumann ${ }^{3}$.

Definition 2.1. For each integrable set-valued random variable $F$, which means $S(F) \neq \emptyset$ has finite expectation, the Aumann integral of $F$, denoted by $E[F]$, is defined by

$$
E[F]=\left\{\int_{\Omega} f d P: f \in S_{F}\right\},
$$

where $S_{F}=\{f: f(\omega) \in F(\omega)$ a.s. $(P)$ and $f$ is integrable $\}$ is called the selection of set-valued random variable $F, \int_{\Omega} f d P$ is the usual Bochner integral.

The properties of the expectation of set-valued random variables have been discussed ${ }^{11,18}$. However, since the space of subsets of $\mathscr{X}$ is not a linear space with respect to the addition and multiplication, the minus between two sets is difficult to define. Thus, extending the important notions of variance and covariance to the case of set-valued random variables is not a trivial task. Yang and $\mathrm{Li}^{27}$ proposed to define the variance and covariance using the $D_{p}$ metric on $\mathscr{U}\left[\Omega, \mathbf{K}_{k c}\left(\mathbb{R}^{d}\right)\right]$, based on the fact that the support functions of sets are subtractive.
Definition 2.2. For each set-valued random variable $F \in \mathscr{U}\left[\Omega, \boldsymbol{K}_{k c}(\mathscr{X})\right]$, the variance of $F$, denoted by $\operatorname{Var}(F)$, is defined as

$$
\begin{aligned}
& \operatorname{Var}(F)=\left[D_{2}(F, E(F))\right]^{2} \\
= & E\left\{\int_{S^{*}}\left[s\left(x^{*}, F(\omega)\right)-s\left(x^{*}, E(F(\omega))\right)\right]^{2} d \mu\right\} .
\end{aligned}
$$

For two set-valued random variables $F_{1}, F_{2} \in$ $\mathscr{U}\left[\Omega, K_{k c}(\mathscr{X})\right]$, the covariance of $F_{1}$ and $F_{2}$, denoted by $\operatorname{Cov}\left(F_{1}, F_{2}\right)$, is defined as

$$
\begin{aligned}
\operatorname{Cov}\left(F_{1}, F_{2}\right)= & E\left\{\int_{S^{*}}\left[s\left(x^{*}, F_{1}(\omega)\right)-s\left(x^{*}, E\left(F_{1}\right)\right)\right]\right. \\
& {\left.\left[s\left(x^{*}, F_{2}(\omega)\right)-s\left(x^{*}, E\left(F_{2}\right)\right)\right] d \mu\right\} . }
\end{aligned}
$$

The correlation coefficient of $F_{1}$ and $F_{2}$, denoted by $\rho\left(\mathrm{F}_{1}, \mathrm{~F}_{2}\right)$, is defined as

$$
\rho\left(F_{1}, F_{2}\right)=\frac{\operatorname{Cov}\left(F_{1}, F_{2}\right)}{\sqrt{\operatorname{Var}\left(F_{1}\right) \cdot \operatorname{Var}\left(F_{2}\right)}} .
$$

The variance and covariance of set-valued random variables have the following properties. The proofs of Theorems 2.3-2.5 can be found in Wang and $\mathrm{Li}^{25}$.

Theorem 2.3. The variance $\operatorname{Var}(F)$ of $F \in$ $\mathscr{U}\left[\Omega, \boldsymbol{K}_{k c}(\mathscr{X})\right]$ has the following properties:

(1) $\operatorname{Var}(C)=0$ for any constant $C \in \boldsymbol{K}_{k c}(\mathscr{X})$.

(2) $\operatorname{Var}(a F)=a^{2} \operatorname{Var}(F)$ for any $a \geqslant 0$.

(3) $\operatorname{Var}\left(F_{1}+F_{2}\right)=\operatorname{Var}\left(F_{1}\right)+2 \operatorname{Cov}\left(F_{1}, F_{2}\right)+$ $\operatorname{Var}\left(F_{2}\right)$.

(4) (Chebyshev Inequality) $P\left(d_{2}(F, E(F)) \geqslant\right.$ $\varepsilon)) \leqslant \operatorname{Var}(F) / \varepsilon^{2}$, for any $\varepsilon>0$.

Theorem 2.4. The covariance $\operatorname{Cov}\left(F_{1}, F_{2}\right)$ of $F_{1}, F_{2} \in \mathscr{U}\left[\Omega, \boldsymbol{K}_{k c}(\mathscr{X})\right]$ has the following properties:

(1) $\operatorname{Cov}\left(a F_{1}, F_{2}\right)=\operatorname{Cov}\left(F_{1}, a F_{2}\right)=a \operatorname{Cov}\left(F_{1}, F_{2}\right)$ for any $a \geqslant 0$.

(2) $\operatorname{Cov}\left(F_{1}+F_{2}, F_{3}\right)=\operatorname{Cov}\left(F_{1}, F_{3}\right)+$ $\operatorname{Cov}\left(F_{2}, F_{3}\right), \quad \operatorname{Cov}\left(F_{1}, F_{2}+F_{3}\right)=\operatorname{Cov}\left(F_{1}, F_{2}\right)+$ $\operatorname{Cov}\left(F_{1}, F_{3}\right)$. 
Theorem 2.5. For any two interval-valued random variables $X_{1}(\omega)=\left[a_{1}(\omega), b_{1}(\omega)\right]=$ $\left(c_{1}(\omega) ; r_{1}(\omega)\right), X_{2}(\omega)=\left[a_{2}(\omega), b_{2}(\omega)\right]=$ $\left(c_{2}(\omega) ; r_{2}(\omega)\right)$, where $c_{i}(\omega)=\left(a_{i}(\omega)+b_{i}(\omega)\right) / 2$ is the center and $r_{i}(\omega)=\left(b_{i}(\omega)-a_{i}(\omega)\right) / 2$ is the radius of $X_{i}(\omega), i=1,2$, their covariance matrix is

$$
\begin{aligned}
& \operatorname{Cov}\left(X_{1}(\omega), X_{2}(\omega)\right) \\
= & \operatorname{Cov}\left(a_{1}(\omega), a_{2}(\omega)\right)+\operatorname{Cov}\left(b_{1}(\omega), b_{2}(\omega)\right) \\
= & 2 \operatorname{Cov}\left(c_{1}(\omega), c_{2}(\omega)\right)+2 \operatorname{Cov}\left(r_{1}(\omega), r_{2}(\omega)\right) .
\end{aligned}
$$

Remark 2.3. For an interval-valued random variable $F \in \mathscr{U}\left[\Omega, \mathbf{K}_{k c}\left(\mathbb{R}^{1}\right)\right]$, denoted as $F(\omega)=$ $[f(\omega), g(\omega)]=(c(\omega) ; r(\omega))$, where $f(\omega), g(\omega)$ are real-valued random variables and $f(\omega) \leqslant g(\omega)$, $c(\omega)=(f(\omega)+g(\omega)) / 2, r(\omega)=(g(\omega)-f(\omega)) / 2$, by the definition of Aumann integral and variance of set-valued random variables, we have

$E(F(\omega))=[E(f(\omega)), E(g(\omega))]=(E(c(\omega)) ; E(r(\omega)))$

and

$$
\begin{aligned}
& \operatorname{Var}(\mathrm{F}(\omega)) \\
= & E\left(|f(\omega)-E(f)|^{2}\right)+E\left(|g(\omega)-E(g)|^{2}\right) \\
= & E\left(|c(\omega)-E(c)-(r(\omega)-E(r))|^{2}\right) \\
& +E\left(|c(\omega)-E(c)+(r(\omega)-E(r))|^{2}\right) .
\end{aligned}
$$

For interval-valued random variables $F_{1}, F_{2} \in$ $\mathscr{U}\left[\Omega, \mathbf{K}_{k c}\left(\mathbb{R}^{1}\right)\right]$,

$$
\begin{aligned}
& \operatorname{Cov}\left(\mathrm{F}_{1}(\omega), \mathrm{F}_{2}(\omega)\right) \\
= & E\left(\left|f_{1}(\omega)-E\left(f_{1}\right)\right|\left|f_{2}(\omega)-E\left(f_{2}\right)\right|\right) \\
& +E\left(\left|g_{1}(\omega)-E\left(g_{1}\right)\right|\left|g_{2}(\omega)-E\left(g_{2}\right)\right|\right) \\
= & E\left(\left|c_{1}(\omega)-E\left(c_{1}\right)-\left(r_{1}(\omega)-E\left(r_{1}\right)\right)\right|\right. \\
& \left.\left|c_{2}(\omega)-E\left(c_{2}\right)-\left(r_{2}(\omega)-E\left(r_{2}\right)\right)\right|\right) \\
& +E\left(\left|c_{1}(\omega)-E\left(c_{1}\right)+\left(r_{1}(\omega)-E\left(r_{1}\right)\right)\right|\right. \\
& \left.\left|c_{2}(\omega)-E\left(c_{2}\right)+\left(r_{2}(\omega)-E\left(r_{2}\right)\right)\right|\right) .
\end{aligned}
$$

\section{Interval-Valued Linear Model and Least Square Estimation}

In this section, we consider an interval-valued linear model with the following general form

$$
E(y)=X \beta,
$$

where $y=\left(y_{1}, y_{2}, \cdots, y_{n}\right)^{T}$ is an $n$-dimensional vector of interval-valued observations, $X=\left(x_{i j}\right)_{i=1, j=1}^{n, p}$ is an $n \times p$ design matrix, $\beta=\left(\beta_{1}, \beta_{2}, \cdots, \beta_{p}\right)^{T}$ is a $p \times 1$ interval-valued parameter vector.

Definition 3.1. If $\left(y_{i} ; x_{i 1}, x_{i 2}, \cdots, x_{i p}\right), i=1,2, \cdots, n$ are $n$ independent observations of interval-valued linear model (1), the least square estimator (LSE) of unknown parameters $\beta$ is the estimator which minimizes $d_{2}(y, X \beta)$.

From the definition of the $d_{p}$ metric, we have

$$
\begin{aligned}
& d_{2}^{2}(y, X \beta) \\
= & \sum_{i=1}^{n} d_{2}^{2}\left(y_{i}, x_{i 1} \beta_{1}+x_{i 2} \beta_{2}+\cdots,+x_{i p} \beta_{p}\right) \\
= & \sum_{i=1}^{n}\left[\left(c_{y_{i}}-x_{i 1} c_{\beta_{1}}-\cdots-x_{i p} c_{\beta_{p}}\right)\right. \\
& \left.-\left(r_{y_{i}}-\left|x_{i 1}\right| r_{\beta_{1}}-\cdots-\left|x_{i p}\right| r_{\beta_{p}}\right)\right]^{2} \\
& +\sum_{i=1}^{n}\left[\left(c_{y_{i}}-x_{i 1} c_{\beta_{1}}-\cdots-x_{i p} c_{\beta_{p}}\right)\right. \\
& \left.+\left(r_{y_{i}}-\left|x_{i 1}\right| r_{\beta_{1}}-\cdots-\left|x_{i p}\right| r_{\beta_{p}}\right)\right]^{2} \\
= & 2 \sum_{i=1}^{n}\left[\left(c_{y_{i}}-x_{i 1} c_{\beta_{1}}-\cdots-x_{i p} c_{\beta_{p}}\right)^{2}\right. \\
& \left.+\left(r_{y_{i}}-\left|x_{i 1}\right| r_{\beta_{1}}-\cdots-\left|x_{i p}\right| r_{\beta_{p}}\right)^{2}\right],
\end{aligned}
$$

where $c_{A}$ and $r_{A}$ stand for the center and radius of interval $A$ respectively. This is a quadratic function of $c_{\beta_{1}}, \cdots, c_{\beta_{p}}, r_{\beta_{1}}, \cdots, r_{\beta_{p}}$ and $d_{2}^{2}(y, X \beta) \geqslant 0$, so there exists a minimum value, which satisfies

$$
\frac{\partial d_{2}^{2}(y, X \beta)}{\partial c_{\beta_{j}}}=0, \frac{\partial d_{2}^{2}(y, X \beta)}{\partial r_{\beta_{j}}}=0, j=1,2, \cdots, p,
$$

that is

$$
\left\{\begin{array}{l}
\sum_{i=1}^{n}\left(c_{y_{i}}-x_{i 1} c_{\beta_{1}}-\cdots-x_{i p} c_{\beta_{p}}\right)\left(-x_{i j}\right)=0 \\
\sum_{i=1}^{n}\left(r_{y_{i}}-\left|x_{i 1}\right| r_{\beta_{1}}-\cdots-\left|x_{i p}\right| r_{\beta_{p}}\right)\left(-x_{i j}\right)=0,
\end{array}\right.
$$

for $j=1,2, \cdots, p$. Rewriting these equations in matrix form, we get

$$
\left\{\begin{array}{l}
X^{T} c_{y}=X^{T} X c_{\beta} \\
|X|^{T} r_{y}=|X|^{T}|X| r_{\beta}
\end{array}\right.
$$

where $|X|=\left(\left|x_{i j}\right|\right)_{i=1, j=1}^{n, p}$.

We conclude the above discussions by the following theorem. 
Theorem 3.1. If $\operatorname{rank}(X)=\operatorname{rank}(|X|)=p$, the LSE of the interval-valued linear model (1), denoted as $\widehat{\beta}_{L S}$, is unique and

$$
\widehat{\beta}_{L S}=\left(\left(X^{T} X\right)^{-1} X^{T} c_{y} ;\left(|X|^{T}|X|\right)^{-1}|X|^{T} r_{y}\right) .
$$

Moreover, we may obtain following theorems about the LSE $\widehat{\beta}_{L S}$.

Theorem 3.2. The LSE $\widehat{\beta}_{L S}$ is an unbiased estimator of $\beta$.
Proof Since $E(y)=X \beta=\left(X c_{\beta} ; X r_{\beta}\right)$, we have

$$
\begin{aligned}
& E\left(\widehat{\beta}_{L S}\right) \\
= & E\left(\left(X^{T} X\right)^{-1} X^{T} c_{y} ;\left(|X|^{T}|X|\right)^{-1}|X|^{T} r_{y}\right) \\
= & \left(\left(X^{T} X\right)^{-1} X^{T} E\left(c_{y}\right) ;\left(|X|^{T}|X|\right)^{-1}|X|^{T} E\left(r_{y}\right)\right) \\
= & \left(\left(X^{T} X\right)^{-1} X^{T} X c_{\beta} ;\left(|X|^{T}|X|\right)^{-1}|X|^{T}|X| r_{\beta}\right) \\
= & \left(c_{\beta} ; r_{\beta}\right)=\beta .
\end{aligned}
$$

Theorem 3.3. If $E(y)=X \beta, \quad \operatorname{rank}(X)=$ $\operatorname{rank}(|X|)=p$ and $\operatorname{Cov}\left(c_{y}\right)=\sigma_{1}^{2} I_{n}, \operatorname{Cov}\left(r_{y}\right)=\sigma_{2}^{2} I_{n}$, then the covariance matrix of $\widehat{\beta}_{L S}$ is

$$
\operatorname{Cov}\left(\widehat{\beta}_{L S}\right)=2 \sigma_{1}^{2}\left(X^{T} X\right)^{-1}+2 \sigma_{2}^{2}\left(|X|^{T}|X|\right)^{-1} .
$$

Proof By Theorem 2.5, we obtain

$$
\begin{aligned}
& \operatorname{Cov}\left(\widehat{\beta}_{L S}^{(i)}, \widehat{\beta}_{L S}^{(j)}\right) \\
= & \operatorname{Cov}\left(\left(\left[\left(X^{T} X\right)^{-1} X^{T}\right]_{(i)}^{T} c_{y} ;\left[\left(|X|^{T}|X|\right)^{-1}|X|^{T}\right]_{(i)}^{T} r_{y}\right),\left(\left[\left(X^{T} X\right)^{-1} X^{T}\right]_{(j)}^{T} c_{y} ;\left[\left(|X|^{T}|X|\right)^{-1}|X|^{T}\right]_{(j)}^{T} r_{y}\right)\right) \\
= & 2 \operatorname{Cov}\left(\left[\left(X^{T} X\right)^{-1} X^{T}\right]_{(i)}^{T} c_{y},\left[\left(X^{T} X\right)^{-1} X^{T}\right]_{(j)}^{T} c_{y}\right)+2 \operatorname{Cov}\left(\left[\left(|X|^{T}|X|\right)^{-1}|X|^{T}\right]_{(i)}^{T} r_{y},\left[\left(|X|^{T}|X|\right)^{-1}|X|^{T}\right]_{(j)}^{T} r_{y}\right) \\
= & 2\left[\left(X^{T} X\right)^{-1} X^{T}\right]_{(i)}^{T} \operatorname{Cov}\left(c_{y}\right)\left[\left(X^{T} X\right)^{-1} X^{T}\right]_{(j)}+2\left[\left(|X|^{T}|X|\right)^{-1}|X|^{T}\right]_{(i)}^{T} \operatorname{Cov}\left(r_{y}\right)\left[\left(|X|^{T}|X|\right)^{-1}|X|^{T}\right]_{(j)},
\end{aligned}
$$

where $\widehat{\beta}_{L S}^{(i)}$ represents the $i$-th element of vector $\widehat{\beta}_{L S}$ and $A_{(i)}$ stands for the $i$-th line of matrix $A$. Therefore,

$$
\begin{aligned}
& \operatorname{Cov}\left(\widehat{\beta}_{L S}\right) \\
= & 2\left(X^{T} X\right)^{-1} X^{T} \operatorname{Cov}\left(c_{y}\right) X\left(X^{T} X\right)^{-1} \\
& +2\left(|X|^{T}|X|\right)^{-1}|X|^{T} \operatorname{Cov}\left(r_{y}\right)|X|\left(|X|^{T}|X|\right)^{-1} \\
= & 2 \sigma_{1}^{2}\left(X^{T} X\right)^{-1}+2 \sigma_{2}^{2}\left(|X|^{T}|X|\right)^{-1} .
\end{aligned}
$$

\section{Best Linear Unbiased and Binary Linear Unbiased Estimation}

\subsection{Best Linear Unbiased Estimation}

Given $n$ interval-valued data from the intervalvalued linear model (1), $y_{i}=\left[a_{y_{i}}, b_{y_{i}}\right]=\left(c_{y_{i}} ; r_{y_{i}}\right)$ for $i=1,2, \cdots, n$, the best linear unbiased estimator is a linear combination of $y_{1}, y_{2}, \cdots, y_{n}$,

$$
\widehat{\beta}_{j}=\lambda_{j 1} y_{1}+\lambda_{j 2} y_{2}+\cdots+\lambda_{j n} y_{n} \doteq \lambda_{j}^{T} y,
$$

$j=1,2, \cdots, p$, and the estimation is unbiased, that is,

$$
E\left(\widehat{\beta}_{j}\right)=\beta_{j} .
$$

Let $\beta_{j}=\left[a_{\beta_{j}}, b_{\beta_{j}}\right]=\left(c_{\beta_{j}} ; r_{\beta_{j}}\right)$. By (1) and (4), we have

$$
\begin{aligned}
E\left(\widehat{\beta}_{j}\right) & =\lambda_{j}^{T} E(y)=\lambda_{j}^{T}\left(X c_{\beta} ;|X| r_{\beta}\right) \\
& =\left(\lambda_{j}^{T} X c_{\beta} ;\left|\lambda_{j}\right|^{T}|X| r_{\beta}\right),
\end{aligned}
$$

where $\left|\lambda_{j}\right|=\left(\left|\lambda_{j 1}\right|,\left|\lambda_{j 2}\right|, \cdots,\left|\lambda_{j n}\right|\right)^{T}$. Therefore we obtain

$$
E(\widehat{\beta})=\left(\Lambda X c_{\beta} ;|\Lambda||X| r_{\beta}\right),
$$


where

$$
\Lambda=\left(\begin{array}{c}
\lambda_{1}^{T} \\
\lambda_{2}^{T} \\
\vdots \\
\lambda_{p}^{T}
\end{array}\right)=\left(\begin{array}{cccc}
\lambda_{11} & \lambda_{12} & \cdots & \lambda_{1 n} \\
\lambda_{21} & \lambda_{22} & \cdots & \lambda_{2 n} \\
\cdots & \cdots & \cdots & \cdots \\
\lambda_{p 1} & \lambda_{p 2} & \cdots & \lambda_{p n}
\end{array}\right)
$$

and

$$
|\Lambda|=\left(\begin{array}{c}
\left|\lambda_{1}\right|^{T} \\
\left|\lambda_{2}\right|^{T} \\
\vdots \\
\left|\lambda_{p}\right|^{T}
\end{array}\right)=\left(\begin{array}{cccc}
\left|\lambda_{11}\right| & \left|\lambda_{12}\right| & \ldots & \left|\lambda_{1 n}\right| \\
\left|\lambda_{21}\right| & \left|\lambda_{22}\right| & \ldots & \left|\lambda_{2 n}\right| \\
\cdots & \ldots & \ldots & \ldots \\
\left|\lambda_{p 1}\right| & \left|\lambda_{p 2}\right| & \cdots & \left|\lambda_{p n}\right|
\end{array}\right)
$$

On the other hand, since $\widehat{\beta}$ is unbiased,

$$
E(\widehat{\beta})=\left(c_{\beta} ; r_{\beta}\right) .
$$

Therefore, by (5) and (6), we have

$$
\Lambda X=I_{p}, \quad|\Lambda||X|=I_{p} .
$$

Unfortunately, the solution of (7) does not exist in general. For the case $p>1$, we consider the interval-valued linear regression model as an example:

$$
E(y)=\beta_{1}+\beta_{2} X_{2},
$$

where $X_{2}=\left(x_{12}, x_{22}, \cdots, x_{n 2}\right)^{T}$. In this case,

$$
\begin{gathered}
\Lambda=\left(\begin{array}{cccc}
\lambda_{11} & \lambda_{12} & \cdots & \lambda_{1 n} \\
\lambda_{21} & \lambda_{22} & \cdots & \lambda_{2 n}
\end{array}\right), \\
|\Lambda|=\left(\begin{array}{llll}
\left|\lambda_{11}\right| & \left|\lambda_{12}\right| & \cdots & \left|\lambda_{1 n}\right| \\
\left|\lambda_{21}\right| & \left|\lambda_{22}\right| & \cdots & \left|\lambda_{2 n}\right|
\end{array}\right)
\end{gathered}
$$

and

$$
X=\left(\begin{array}{cccc}
1 & 1 & \cdots & 1 \\
x_{21} & x_{22} & \cdots & x_{2 n}
\end{array}\right)^{T},
$$

then the second equation of (7) is

$$
\begin{aligned}
& \sum_{i=1}^{n}\left|\lambda_{1 i}\right|=1, \quad \sum_{i=1}^{n}\left|\lambda_{1 i}\right|\left|x_{2 i}\right|=0, \\
& \sum_{i=1}^{n}\left|\lambda_{2 i}\right|=0, \quad \sum_{i=1}^{n}\left|\lambda_{2 i}\right|\left|x_{2 i}\right|=1 .
\end{aligned}
$$

It is obvious that these equations are contradictory. For the case $p=1$,

$$
E(y)=\left(\begin{array}{c}
x_{11} \\
x_{21} \\
\vdots \\
x_{n 1}
\end{array}\right) \beta_{1},
$$

then (7) becomes

$$
\sum_{i=1}^{n} \lambda_{1 i} x_{i 1}=1, \quad \sum_{i=1}^{n}\left|\lambda_{1 i}\right|\left|x_{i 1}\right|=1 .
$$

Therefore, a linear unbiased estimator exists only if $x_{i 1} \geqslant 0, i=1,2, \cdots, n$.

\subsection{Best Binary Linear Unbiased Estimation}

From the above discussions, we know that, for the interval-valued linear model (1), the best linear unbiased estimator does not exist in general, which is a major difference with the traditional linear model. However, for the interval-valued linear model, we can introduce a new type of estimation: the binary best linear unbiased estimation, which has some interesting statistical properties.

Definition 4.1. The binary linear combination of interval-valued data $y_{i}=\left[a_{y_{i}}, b_{y_{i}}\right]=\left(c_{y_{i}} ; r_{y_{i}}\right), i=$ $1,2, \cdots, n$ with coefficient $k_{i}, l_{i}\left(l_{i} \geqslant 0\right)$ is defined as

$$
\sum_{i=1}^{n}\left(k_{i} c_{y_{i}} ; l_{i} r_{y_{i}}\right)=\left(\sum_{i=1}^{n} k_{i} c_{y_{i}} ; \sum_{i=1}^{n} l_{i} r_{y_{i}}\right) .
$$

Definition 4.2. An estimator of an interval-valued parameter is called a binary linear estimator, if it is a binary linear combination of interval-valued $o b$ servations. Assume that $\widehat{\theta}$ is a binary linear estimator of interval-valued parameter $\theta$. If $\widehat{\theta}$ is unbiased and, for any binary linear unbiased estimator $\theta^{*}$ of $\theta$,

$$
\operatorname{Var}\left(\theta^{*}\right) \geqslant \operatorname{Var}(\widehat{\theta}),
$$

$\widehat{\theta}$ is called best binary linear unbiased estimator (BBLUE) of $\theta$.

If $\theta$ is a $p$-dimensional vector of intervalvalued parameter, $\operatorname{Var}\left(\theta^{*}\right) \geqslant \operatorname{Var}(\widehat{\theta})$ in this definition means that $\operatorname{Cov}\left(\theta^{*}\right)-\operatorname{Cov}(\widehat{\theta})$ is a nonnegative definite matrix. 
Theorem 4.1. If $E(y)=X \beta, \quad \operatorname{rank}(X)=$ $\operatorname{rank}(|X|)=p$ and $\operatorname{Cov}\left(\mathrm{c}_{\mathrm{y}}\right)=\sigma_{1}^{2} \mathrm{I}_{\mathrm{n}}, \operatorname{Cov}\left(\mathrm{r}_{\mathrm{y}}\right)=\sigma_{2}^{2} \mathrm{I}_{\mathrm{n}}$, then the LSE $\widehat{\beta}_{L S}$ is the unique BBLUE.

Proof By Theorem 3.2,

$$
\widehat{\beta}_{L S}=\left(\left(X^{T} X\right)^{-1} X^{T} c_{y} ;\left(|X|^{T}|X|\right)^{-1}|X|^{T} r_{y}\right)
$$

is an unbiased estimator of $\beta$, and binary linearity of $\widehat{\beta}_{L S}$ is obvious. Therefore, we just need to prove that the covariance matrix of $\widehat{\beta}_{L S}$ is the minimum one among all binary linear unbiased estimators.

Assume that

$$
\varphi_{j}(y)=\sum_{i=1}^{n}\left(k_{j i}^{*} c_{y_{i}} ; l_{j i}^{*} r_{y_{i}}\right), \quad l_{j i}^{*} \geqslant 0
$$

is a binary linear unbiased estimate of $\beta_{j}$, then

$$
\varphi(y)=\left(k^{*} c_{y} ; l^{*} r_{y}\right)
$$

is a binary linear unbiased estimator of $\beta$, where

$$
k^{*}=\left(\begin{array}{cccc}
k_{11}^{*} & k_{12}^{*} & \cdots & k_{1 n}^{*} \\
k_{21}^{*} & k_{22}^{*} & \cdots & k_{2 n}^{*} \\
\cdots & \cdots & \cdots & \cdots \\
k_{p 1}^{*} & k_{p 2}^{*} & \cdots & k_{p n}^{*}
\end{array}\right)
$$

and

$$
l^{*}=\left(\begin{array}{cccc}
l_{11}^{*} & l_{12}^{*} & \cdots & l_{1 n}^{*} \\
l_{21}^{*} & l_{22}^{*} & \cdots & l_{2 n}^{*} \\
\cdots & \cdots & \cdots & \cdots \\
l_{p 1}^{*} & l_{p 2}^{*} & \cdots & l_{p n}^{*}
\end{array}\right),
$$

and $l^{*} \geqslant 0$. By the unbiasedness of $\varphi(y)$,

$$
\begin{aligned}
E(\varphi(y)) & =\left(k^{*} E\left(c_{y}\right) ; l^{*} E\left(r_{y}\right)\right)=\left(k^{*} X c_{\beta} ; l^{*}|X| r_{\beta}\right) \\
& =\left(c_{\beta} ; r_{\beta}\right), \forall c_{\beta}, r_{\beta} \in \mathbb{R}^{p} .
\end{aligned}
$$

Hence we have

$$
k^{*} X=I_{p}, l^{*}|X|=I_{p} .
$$

By Theorem 2.5,

$$
\begin{aligned}
& \operatorname{Cov}\left(\varphi_{i}(y), \varphi_{j}(y)\right) \\
= & \operatorname{Cov}\left(\left(\sum_{m=1}^{n} k_{i m}^{*} c_{y_{m}} ; \sum_{m=1}^{n} l_{i m}^{*} r_{y_{m}}\right),\right. \\
& \left.\left(\sum_{m=1}^{n} k_{j m}^{*} c_{y_{m}} ; \sum_{m=1}^{n} l_{j m}^{*} r_{y_{m}}\right)\right) \\
= & E\left\{\left[\left(k^{*}\right)_{(i)}^{T} c_{y}+\left(l^{*}\right)_{(i)}^{T} r_{y}-\left(c_{\beta_{i}}+r_{\beta_{i}}\right)\right]\right. \\
& {\left.\left[\left(k^{*}\right)_{(j)}^{T} c_{y}+\left(l^{*}\right)_{(j)}^{T} r_{y}-\left(c_{\beta_{j}}+r_{\beta_{j}}\right)\right]\right\} } \\
& +E\left\{\left[\left(k^{*}\right)_{(i)}^{T} c_{y}-\left(l^{*}\right)_{(i)}^{T} r_{y}-\left(c_{\beta_{i}}-r_{\beta_{i}}\right)\right]\right. \\
& {\left.\left[\left(k^{*}\right)_{(j)}^{T} c_{y}-\left(l^{*}\right)_{(j)}^{T} r_{y}-\left(c_{\beta_{j}}-r_{\beta_{j}}\right)\right]\right\} . }
\end{aligned}
$$

Then, we obtain

$$
\begin{aligned}
& \operatorname{Cov}(\varphi(y)) \\
= & E\left\{\left[k^{*} c_{y}+l^{*} r_{y}-\left(c_{\beta}+r_{\beta}\right)\right]\left[k^{*} c_{y}+l^{*} r_{y}-\left(c_{\beta}+r_{\beta}\right)\right]^{T}\right\}+E\left\{\left[k^{*} c_{y}-l^{*} r_{y}-\left(c_{\beta}-r_{\beta}\right)\right]\left[k^{*} c_{y}-l^{*} r_{y}-\left(c_{\beta}-r_{\beta}\right)\right]^{T}\right\} \\
= & E\left\{\left[k^{*} c_{y}+l^{*} r_{y}-\left(\left(X^{T} X\right)^{-1} X^{T} c_{y}+\left(|X|^{T}|X|\right)^{-1}|X|^{T} r_{y}\right)\right]\left[k^{*} c_{y}+l^{*} r_{y}-\left(\left(X^{T} X\right)^{-1} X^{T} c_{y}+\left(|X|^{T}|X|\right)^{-1}|X|^{T} r_{y}\right)\right]^{T}\right\} \\
& \left.+\operatorname{Cov}\left(\left(X^{T} X\right)^{-1} X^{T} c_{y}+\left(|X|^{T}|X|\right)^{-1}|X|^{T} r_{y}\right)\right) \\
& \left.+E\left\{\left[k^{*} c_{y}+l^{*} r_{y}-\left(\left(X^{T} X\right)^{-1} X^{T} c_{y}+\left(|X|^{T}|X|\right)^{-1}|X|^{T} r_{y}\right)\right]\left[\left(X^{T} X\right)^{-1} X^{T} c_{y}+\left(|X|^{T}|X|\right)^{-1}|X|^{T} r_{y}\right)-\left(c_{\beta}+r_{\beta}\right)\right]^{T}\right\} \\
& \left.+E\left\{\left[\left(X^{T} X\right)^{-1} X^{T} c_{y}+\left(|X|^{T}|X|\right)^{-1}|X|^{T} r_{y}\right)-\left(c_{\beta}+r_{\beta}\right)\right]\left[k^{*} c_{y}+l^{*} r_{y}-\left(\left(X^{T} X\right)^{-1} X^{T} c_{y}+\left(|X|^{T}|X|\right)^{-1}|X|^{T} r_{y}\right)\right]^{T}\right\} \\
& +E\left\{\left[k^{*} c_{y}-l^{*} r_{y}-\left(\left(X^{T} X\right)^{-1} X^{T} c_{y}-\left(|X|^{T}|X|\right)^{-1}|X|^{T} r_{y}\right)\right]\left[k^{*} c_{y}-l^{*} r_{y}-\left(\left(X^{T} X\right)^{-1} X^{T} c_{y}-\left(|X|^{T}|X|\right)^{-1}|X|^{T} r_{y}\right)\right]^{T}\right\} \\
& \left.+\operatorname{Cov}\left(\left(X^{T} X\right)^{-1} X^{T} c_{y}-\left(|X|^{T}|X|\right)^{-1}|X|^{T} r_{y}\right)\right) \\
& \left.+E\left\{\left[k^{*} c_{y}-l^{*} r_{y}-\left(\left(X^{T} X\right)^{-1} X^{T} c_{y}-\left(|X|^{T}|X|\right)^{-1}|X|^{T} r_{y}\right)\right]\left[\left(X^{T} X\right)^{-1} X^{T} c_{y}-\left(|X|^{T}|X|\right)^{-1}|X|^{T} r_{y}\right)-\left(c_{\beta}-r_{\beta}\right)\right]^{T}\right\} \\
& \left.+E\left\{\left[\left(X^{T} X\right)^{-1} X^{T} c_{y}-\left(|X|^{T}|X|\right)^{-1}|X|^{T} r_{y}\right)-\left(c_{\beta}-r_{\beta}\right)\right]\left[k^{*} c_{y}-l^{*} r_{y}-\left(\left(X^{T} X\right)^{-1} X^{T} c_{y}-\left(|X|^{T}|X|\right)^{-1}|X|^{T} r_{y}\right)\right]^{T}\right\} .
\end{aligned}
$$


Since

and

$$
E\left[k^{*} c_{y}-l^{*} r_{y}-\left(\left(X^{T} X\right)^{-1} X^{T} c_{y}-\left(|X|^{T}|X|\right)^{-1}|X|^{T} r_{y}\right)\right]=0
$$

$E\left[k^{*} c_{y}+l^{*} r_{y}-\left(\left(X^{T} X\right)^{-1} X^{T} c_{y}+\left(|X|^{T}|X|\right)^{-1}|X|^{T} r_{y}\right)\right]=0 \quad$ we have the following equalities

$$
\begin{aligned}
& \left.E\left\{\left[k^{*} c_{y}+l^{*} r_{y}-\left(\left(X^{T} X\right)^{-1} X^{T} c_{y}+\left(|X|^{T}|X|\right)^{-1}|X|^{T} r_{y}\right)\right]\left[\left(X^{T} X\right)^{-1} X^{T} c_{y}+\left(|X|^{T}|X|\right)^{-1}|X|^{T} r_{y}\right)-\left(c_{\beta}+r_{\beta}\right)\right]^{T}\right\} \\
& \left.+E\left\{\left[k^{*} c_{y}-l^{*} r_{y}-\left(\left(X^{T} X\right)^{-1} X^{T} c_{y}-\left(|X|^{T}|X|\right)^{-1}|X|^{T} r_{y}\right)\right]\left[\left(X^{T} X\right)^{-1} X^{T} c_{y}-\left(|X|^{T}|X|\right)^{-1}|X|^{T} r_{y}\right)-\left(c_{\beta}-r_{\beta}\right)\right]^{T}\right\} \\
= & \operatorname{Cov}\left(k^{*} c_{y}+l^{*} r_{y}-\left(\left(X^{T} X\right)^{-1} X^{T} c_{y}+\left(|X|^{T}|X|\right)^{-1}|X|^{T} r_{y}\right),\left(X^{T} X\right)^{-1} X^{T} c_{y}+\left(|X|^{T}|X|\right)^{-1}|X|^{T} r_{y}\right) \\
& +\operatorname{Cov}\left(k^{*} c_{y}-l^{*} r_{y}-\left(\left(X^{T} X\right)^{-1} X^{T} c_{y}-\left(|X|^{T}|X|\right)^{-1}|X|^{T} r_{y}\right),\left(X^{T} X\right)^{-1} X^{T} c_{y}-\left(|X|^{T}|X|\right)^{-1}|X|^{T} r_{y}\right) \\
= & \operatorname{Cov}\left(\left(k^{*}-\left(X^{T} X\right)^{-1} X^{T}\right) c_{y}+\left(l^{*}-\left(|X|^{T}|X|\right)^{-1}|X|^{T}\right) r_{y},\left(X^{T} X\right)^{-1} X^{T} c_{y}+\left(|X|^{T}|X|\right)^{-1}|X|^{T} r_{y}\right) \\
& +\operatorname{Cov}\left(\left(k^{*}-\left(X^{T} X\right)^{-1} X^{T}\right) c_{y}-\left(l^{*}-\left(|X|^{T}|X|\right)^{-1}|X|^{T}\right) r_{y},\left(X^{T} X\right)^{-1} X^{T} c_{y}-\left(|X|^{T}|X|\right)^{-1}|X|^{T} r_{y}\right) \\
= & 2\left(k^{*}-\left(X^{T} X\right)^{-1} X^{T}\right) \operatorname{Cov}\left(c_{y}\right)\left(\left(X^{T} X\right)^{-1} X^{T}\right)^{T}+2\left(l^{*}-\left(|X|^{T}|X|\right)^{-1}|X|^{T}\right) \operatorname{Cov}\left(r_{y}\right)\left(\left(|X|^{T}|X|\right)^{-1}|X|^{T}\right)^{T} \\
= & 2 \sigma_{1}^{2}\left(k^{*} X-\left(X^{T} X\right)^{-1} X^{T} X\right)\left(X^{T} X\right)^{-1}+2 \sigma_{2}^{2}\left(l^{*}|X|-\left(|X|^{T}|X|\right)^{-1}|X|^{T}|X|\right)\left(|X|^{T}|X|\right)^{-1} \\
= & 0,
\end{aligned}
$$

where the last equality holds due to (8). Hence, we have

$$
\begin{aligned}
& \operatorname{Cov}(\varphi(y)) \\
= & E\left\{\left[k^{*} c_{y}+l^{*} r_{y}-\left(\left(X^{T} X\right)^{-1} X^{T} c_{y}+\left(|X|^{T}|X|\right)^{-1}|X|^{T} r_{y}\right)\right]\left[k^{*} c_{y}+l^{*} r_{y}-\left(\left(X^{T} X\right)^{-1} X^{T} c_{y}+\left(|X|^{T}|X|\right)^{-1}|X|^{T} r_{y}\right)\right]^{T}\right\} \\
& +E\left\{\left[k^{*} c_{y}-l^{*} r_{y}-\left(\left(X^{T} X\right)^{-1} X^{T} c_{y}-\left(|X|^{T}|X|\right)^{-1}|X|^{T} r_{y}\right)\right]\left[k^{*} c_{y}-l^{*} r_{y}-\left(\left(X^{T} X\right)^{-1} X^{T} c_{y}-\left(|X|^{T}|X|\right)^{-1}|X|^{T} r_{y}\right)\right]^{T}\right\} \\
& +\operatorname{Cov}\left(\left(X^{T} X\right)^{-1} X^{T} c_{y}+\left(|X|^{T}|X|\right)^{-1}|X|^{T} r_{y}\right)+\operatorname{Cov}\left(\left(X^{T} X\right)^{-1} X^{T} c_{y}-\left(|X|^{T}|X|\right)^{-1}|X|^{T} r_{y}\right) \\
\geqslant & \operatorname{Cov}\left(\left(X^{T} X\right)^{-1} X^{T} c_{y}+\left(|X|^{T}|X|\right)^{-1}|X|^{T} r_{y}\right)+\operatorname{Cov}\left(\left(X^{T} X\right)^{-1} X^{T} c_{y}-\left(|X|^{T}|X|\right)^{-1}|X|^{T} r_{y}\right) \\
= & \operatorname{Cov}\left(\widehat{\beta}_{L S}\right) .
\end{aligned}
$$

Thus, the LSE $\widehat{\beta}_{L S}$ is BBLUE. Furthermore, $\operatorname{Cov}(\varphi(y))=\operatorname{Cov}\left(\widehat{\beta}_{L S}\right)$ if and only if

$k^{*} c_{y}+l^{*} r_{y}-\left(\left(X^{T} X\right)^{-1} X^{T} c_{y}+\left(|X|^{T}|X|\right)^{-1}|X|^{T} r_{y}\right)=0$,

and

$k^{*} c_{y}-l^{*} r_{y}-\left(\left(X^{T} X\right)^{-1} X^{T} c_{y}-\left(|X|^{T}|X|\right)^{-1}|X|^{T} r_{y}\right)=0$,

a.s., i.e., $\varphi(y)=\widehat{\beta}_{L S}$. Therefore, $\widehat{\beta}_{L S}$ is the unique BBLUE.

Theorem 4.2. If $E(y)=X \beta, \quad \operatorname{rank}(X)=$ $\operatorname{rank}(|X|)=p$ and $\operatorname{Cov}\left(c_{y}\right)=\sigma_{1}^{2} I_{n}, \operatorname{Cov}\left(r_{y}\right)=\sigma_{2}^{2} I_{n}$, then for for all $\alpha \in \mathbb{R}^{p}, \alpha^{T} \widehat{\beta}_{L S}$ is the unique BBLUE of $\alpha^{T} \beta$.
Proof By Theorem 3.2, we have

$$
\begin{aligned}
& E\left(\alpha^{T} \widehat{\beta}_{L S}\right) \\
= & E\left(\alpha^{T}\left(X^{T} X\right)^{-1} X^{T} c_{y} ;|\alpha|^{T}\left(|X|^{T}|X|\right)^{-1}|X|^{T} r_{y}\right) \\
= & \left(\alpha^{T}\left(X^{T} X\right)^{-1} X^{T} X c_{\beta} ;|\alpha|^{T}\left(|X|^{T}|X|\right)^{-1}|X|^{T}|X| r_{\beta}\right) \\
= & \alpha^{T} \beta,
\end{aligned}
$$

which means that

$\alpha^{T} \widehat{\beta}_{L S}=\left(\alpha^{T}\left(X^{T} X\right)^{-1} X^{T} c_{y} ;|\alpha|^{T}\left(|X|^{T}|X|\right)^{-1}|X|^{T} r_{y}\right)$

is a binary linear unbiased estimator of $\alpha^{T} \beta$.

Assume that $\psi(y)$ is a binary linear unbiased estimator of $\alpha^{T} \beta$, denoted as $\psi(y)=\left(k^{T} c_{y} ; l^{T} r_{y}\right)$, 
where $k, l$ are $n$-dimensional vectors and $l \geqslant 0$. Then

$$
\begin{aligned}
& E(\psi(y))=E\left(k^{T} c_{y} ; l^{T} r_{y}\right)=\left(k^{T} X c_{\beta} ; l^{T}|X| r_{\beta}\right) \\
= & \alpha^{T} \beta=\left(\alpha^{T} c_{\beta} ;|\alpha|^{T} r_{\beta}\right), \forall c_{\beta}, r_{\beta} \in \mathbb{R}^{p}
\end{aligned}
$$

Therefore,

$$
k^{T} X=\alpha^{T}, l^{T}|X|=|\alpha|^{T} .
$$

From Remark 2.3,

$$
\begin{aligned}
& \operatorname{Var}(\psi(y))=\operatorname{Var}\left(\left(k^{T} c_{y} ; l^{T} r_{y}\right)\right)=2 \operatorname{Var}\left(k^{T} c_{y}\right)+2 \operatorname{Var}\left(l^{T} r_{y}\right) \\
= & 2 E\left(k^{T} c_{y}-\alpha^{T} c_{\beta}\right)^{2}+2 E\left(l^{T} r_{y}-|\alpha|^{T} c_{\beta}\right)^{2} \\
= & 2 E\left(k^{T} c_{y}-\alpha^{T}\left(X^{T} X\right)^{-1} X^{T} c_{y}+\alpha^{T}\left(X^{T} X\right)^{-1} X^{T} c_{y}-\alpha^{T} c_{\beta}\right)^{2} \\
& +2 E\left(l^{T} r_{y}-|\alpha|^{T}\left(|X|^{T}|X|\right)^{-1}|X|^{T} r_{y}+|\alpha|^{T}\left(|X|^{T}|X|\right)^{-1}|X|^{T} r_{y}-|\alpha|^{T} c_{\beta}\right)^{2} \\
= & 2 E\left[\left(k^{T}-\alpha^{T}\left(X^{T} X\right)^{-1} X^{T}\right) c_{y}\right]^{2}+2 E\left[\left(l^{T}-|\alpha|^{T}\left(|X|^{T}|X|\right)^{-1}|X|^{T}\right) r_{y}\right]^{2}+2 \operatorname{Var}\left(\alpha^{T}\left(X^{T} X\right)^{-1} X^{T} c_{y}\right) \\
& +2 \operatorname{Var}\left(|\alpha|^{T}\left(|X|^{T}|X|\right)^{-1}|X|^{T} r_{y}\right)+4 E\left[\left(k^{T} c_{y}-\alpha^{T}\left(X^{T} X\right)^{-1} X^{T} c_{y}\right)\left(\alpha^{T}\left(X^{T} X\right)^{-1} X^{T} c_{y}-\alpha^{T} c_{\beta}\right)\right] \\
& +4 E\left[\left(l^{T} r_{y}-|\alpha|^{T}\left(|X|^{T}|X|\right)^{-1}|X|^{T} r_{y}\right)\left(|\alpha|^{T}\left(|X|^{T}|X|\right)^{-1}|X|^{T} r_{y}-|\alpha|^{T} r_{\beta}\right)\right] .
\end{aligned}
$$

As $E\left(k^{T} c_{y}-\alpha^{T}\left(X^{T} X\right)^{-1} X^{T} c_{y}\right)=0$ and $E\left(l^{T} r_{y}-|\alpha|^{T}\left(|X|^{T}|X|\right)^{-1}|X|^{T} r_{y}\right)=0$, we have

$$
\begin{aligned}
& E\left[\left(k^{T} c_{y}-\alpha^{T}\left(X^{T} X\right)^{-1} X^{T} c_{y}\right)\right. \\
& \left.\left(\alpha^{T}\left(X^{T} X\right)^{-1} X^{T} c_{y}-\alpha^{T} c_{\beta}\right)\right] \\
= & \operatorname{Cov}\left(k^{T} c_{y}-\alpha^{T}\left(X^{T} X\right)^{-1} X^{T} c_{y}, \alpha^{T}\left(X^{T} X\right)^{-1} X^{T} c_{y}\right) \\
= & \left(k^{T}-\alpha^{T}\left(X^{T} X\right)^{-1} X^{T}\right) \operatorname{Cov}\left(c_{y}\right) X\left(X^{T} X\right)^{-1} \alpha \\
= & \sigma_{1}^{2} k^{T} X\left(X^{T} X\right)^{-1} \alpha-\sigma_{1}^{2} \alpha^{T}\left(X^{T} X\right)^{-1} \alpha \\
= & 0,
\end{aligned}
$$

where the last equality follows from (9). Similarly, we can obtain

$$
\begin{aligned}
& E\left[\left(l^{T} r_{y}-|\alpha|^{T}\left(|X|^{T}|X|\right)^{-1}|X|^{T} r_{y}\right)\right. \\
& \left.\left(|\alpha|^{T}\left(|X|^{T}|X|\right)^{-1}|X|^{T} r_{y}-|\alpha|^{T} r_{\beta}\right)\right]=0 .
\end{aligned}
$$

So we get,

$$
\begin{aligned}
& \operatorname{Var}(\psi(\mathrm{y})) \\
= & 2 E\left[\left(k^{T}-\alpha^{T}\left(X^{T} X\right)^{-1} X^{T}\right) c_{y}\right]^{2} \\
& +2 E\left[\left(l^{T}-|\alpha|^{T}\left(|X|^{T}|X|\right)^{-1}|X|^{T}\right) r_{y}\right]^{2} \\
& +\operatorname{Var}\left(\alpha^{\mathrm{T}} \widehat{\beta}_{\mathrm{LS}}\right) \\
\geqslant & \operatorname{Var}\left(\alpha^{\mathrm{T}} \widehat{\beta}_{\mathrm{LS}}\right) .
\end{aligned}
$$

Furthermore, "=" is tenable if and only if $\left(k^{T}-\alpha^{T}\left(X^{T} X\right)^{-1} X^{T}\right) c_{y}=0$ and $\left(l^{T}-\right.$
$\left.|\alpha|^{T}\left(|X|^{T}|X|\right)^{-1}|X|^{T}\right) r_{y}=0$, a.s., i.e., $\psi(y)=$ $\alpha^{T} \widehat{\beta}_{L S}$. Therefore, $\alpha^{T} \widehat{\beta}_{L S}$ is the unique BBLUE of $\alpha^{T} \beta$.

\section{Simulation Results}

\subsection{Test of Estimation Efficiency}

In this section, we illustrate the interval-valued linear regression model by simulation experiments. Let $\beta_{1}=[1,2]=(1.5 ; 0.5), \beta_{2}=[1.7,2.3]=(2 ; 0.3)$ and

$$
\begin{aligned}
y_{i} & =\beta_{1}+x_{i} \beta_{2}+\varepsilon_{i} \\
& =\left(1.5+2 x_{i}+c_{\varepsilon_{i}} ; 0.5+0.3 x_{i}+r_{\varepsilon_{i}}\right),
\end{aligned}
$$

for $i=1,2, \cdots, n$, where $c_{\varepsilon_{i}}, r_{\varepsilon_{i}}$ are $N\left(0,0.3^{2}\right)$ normal independent random variables, so that $E\left(y_{i}\right)=$ $\beta_{1}+E\left(x_{i}\right) \beta_{2}$. Therefore, we have

$$
E y=E\left(\begin{array}{c}
y_{1} \\
y_{2} \\
\vdots \\
y_{n}
\end{array}\right)=\left(\begin{array}{cc}
1 & x_{1} \\
1 & x_{2} \\
\vdots & \vdots \\
1 & x_{n}
\end{array}\right)\left(\begin{array}{l}
\beta_{1} \\
\beta_{2}
\end{array}\right)=X\left(\begin{array}{c}
\beta_{1} \\
\beta_{2}
\end{array}\right) \text {. }
$$

Firstly, we let the quantity of observations $n$ be $100, x_{i}=0.5+0.01 i, i=1,2, \cdots, 100$. For each repetition of the experiment, we get a LSE $\widehat{\beta}_{L S}$ of 
$\beta_{1}, \beta_{2}$. Figure 1 shows the simulation experiment, in which $\widehat{\beta}_{L S}=([1.06,2.02],[1.66,2.32])^{T}$. In Figure 1 , the points show the simulated data $y_{i}\left(x_{i}\right)=[1,2]+$ $[1.7,2.3] x_{i}+\varepsilon_{i}, x_{i}=0.5+0.01 i, i=1,2, \cdots, 100$ and the two lines represent the interval-valued linear regression function computed by the LSE (3): $y=[1.06,2.02]+[1.66,2.32] x$.

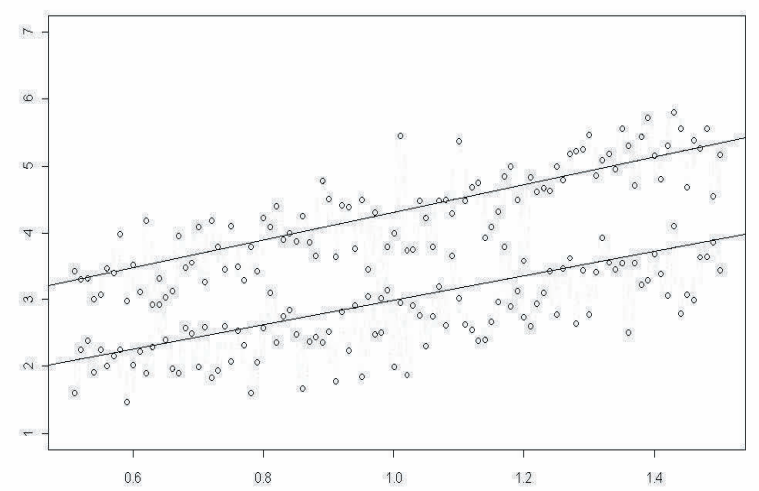

Fig. 1. Simulated data (100 observations) and intervalvalued linear regression function: $y=[1.06,2.02]+$ $[1.66,2.32] x$.

We repeated this experiment (from data generation to parameter estimation) 1000 times. The average value of $\widehat{\beta}_{L S}^{(1)}$ was $[0.9959,1.9964]=$ (1.4961;0.5002), with a sample mean square error (sample MSE) equal to 0.0442 . The average value of $1000 \widehat{\beta}_{L S}^{(2)}$ was $[1.7061,2.3002]=(2.0032 ; 0.2970)$ with a sample MSE is 0.0446 . Here the sample MSE of $\beta$ is defined by $\frac{1}{1000} \sum_{i=1}^{1000} d_{2}^{2}\left(\beta, \widehat{\beta}_{L S}\right)$.

Then, we increased the quantity of observations $n$ to 200 and $300 . X$ was obtained via

$$
\begin{aligned}
& x_{i}=0.5+0.01 i, i=1,2, \cdots, 100, \\
& x_{i}=x_{i-100}, i=101,102, \cdots, 200, \\
& x_{i}=x_{i-200}, i=201,202, \cdots, 300 .
\end{aligned}
$$

Similarly, we obtained estimators of $\widehat{\beta}_{L S}^{(1)}, \widehat{\beta}_{L S}^{(2)}$ by the same method. The results are reported in Tables 1 and 2, which show the average value and the sample MSE of 1000 estimators of $\widehat{\beta}_{L S}^{(1)}$ (the real value is
$[1,2])$ and $\widehat{\beta}_{L S}^{(2)}$ (the real value is $[1.7,2.3]$ ), respectively. We may observe that the sample MSE decreases as the number of observations increases.

Table 1. Average value and sample MSE of $\widehat{\beta}_{L S}^{(1)}$.

\begin{tabular}{|c|c|c|}
\hline & mean value of $\widehat{\beta}_{L S}^{(1)}$ & sample MSE of $\widehat{\beta}_{L S}^{(1)}$ \\
\hline $\mathrm{n}=100$ & {$[0.9959,1.9964]$} & 0.0442 \\
\hline $\mathrm{n}=200$ & {$[1.0029,1.9952]$} & 0.0236 \\
\hline $\mathrm{n}=300$ & {$[1.0025,2.0068]$} & 0.0154 \\
\hline
\end{tabular}

Table 2. Average value and sample MSE of $\widehat{\beta}_{L S}^{(2)}$.

\begin{tabular}{|c|c|c|}
\hline & mean value of $\widehat{\beta}_{L S}^{(2)}$ & sample MSE of $\widehat{\beta}_{L S}^{(2)}$ \\
\hline $\mathrm{n}=100$ & {$[1.7061,2.3002]$} & 0.0446 \\
\hline $\mathrm{n}=200$ & {$[1.7052,2.2990]$} & 0.0220 \\
\hline $\mathrm{n}=300$ & {$[1.6996,2.2960]$} & 0.0142 \\
\hline
\end{tabular}

\subsection{Comparison with Other Models}

When handling point-valued input and intervalvalued output data, an easy and intuitive solution is to fit the left- and right-endpoints, or the centers and the radii, of the interval-valued data by two pointvalued linear models ${ }^{5,14,20}$. As a matter of fact, it is easy to see these two methods are equivalent. As already mentioned in the introduction, a drawback of using two separate point-valued linear models is that it is possible to obtain an interval-valued estimation or forecast result such that the left-endpoint is larger than the right-endpoint (or the radius is negative). In this section, we present the advantage of our model from another point of view via a simulation experiment: comparing the efficiency of the forecast.

We generated the data in the same way as in Section 5.1, with $\beta_{1}=[1,2]=(1.5 ; 0.5), \beta_{2}=$ $[1.7,2.1]=(1.9 ; 0.2)$ and

$$
y_{i}=\beta_{1}+x_{i} \beta_{2}+\varepsilon_{i},
$$

in which $x_{i}=(-3: 0.05: 6)$ and $c_{\varepsilon_{i}}, r_{\varepsilon_{i}}$ are $N\left(0,0.1^{2}\right)$ independent random variables.

We then obtained the following estimates using the LSE for interval-valued linear model (3): $\widehat{\beta}_{L S}=([0.9979,2.0062],[1.7017,2.1000])^{T}$, and the estimated regression function

$$
y=[0.9979,2.0062]+[1.7017,2.1000] x .
$$


In a second step, we fitted $\left(a_{y_{i}}, x_{i}\right)$ and $\left(b_{y_{i}}, x_{i}\right)$, where $a_{y_{i}}$ and $b_{y_{i}}$ are the left- and right-endpoints of $y_{i}$ via two traditional point-valued linear models. Using the LSE for the traditional linear model, we obtained two fitted lines:

$$
\left\{\begin{array}{l}
a_{y}=0.6398+1.8061 x \\
b_{y}=2.3642+1.9956 x
\end{array}\right.
$$

Finally, we generated some new data from (10) and used (11) and (12) to forecast the output respectively. Letting $x_{i}=(-3: 0.2: 6)$ in (10), we obtained the interval-valued output $y_{i}, i=1,2, \cdots, 46$. Next, we substituted $x_{i}=(-3: 0.2: 6)$ back to (11) and (12) and obtained the forecasts of $y_{i}, i=1,2, \cdots, 46$ using the interval-valued LSE (denoted by $\widetilde{y}_{i}$ ) and two endpoints point-valued LSE (denoted by $\widehat{y}_{i}$ ), respectively. The MSE of $\tilde{y}_{i}$ was $\frac{1}{46} \sum_{n=1}^{46} d_{2}^{w}\left(\widetilde{y}_{i}, y_{i}\right)=$ 0.0352 while the MSE of $\widehat{y}_{i}$ was $\frac{1}{46} \sum_{n=1}^{46} d_{2}^{w}\left(\widehat{y}_{i}, y_{i}\right)=$ 0.1290. The box plots in Figure 2 show the median, 25th and 75th percentiles and the extreme data points of the 46 forecasts using interval-valued linear model and using two separate linear models. Since the data were randomly generated, the above procedure (from data generation to forecast) was repeated 30 times. The mean values of the MSEs of the forecasts were 0.0388 for the interval-valued LS estimation and 0.1321 using two endpoints pointvalued LS estimation. Obviously, we can see that the interval-valued linear model is better in the sense that it yields smaller forecasting error.

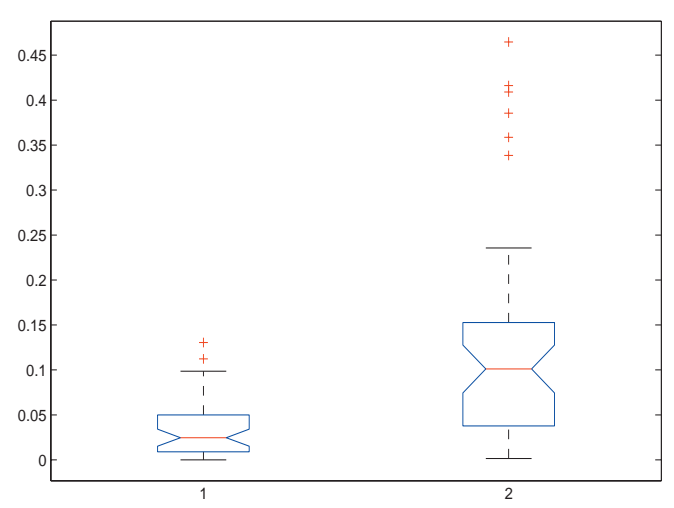

Fig. 2. Box plots of forecasts results using interval-valued linear model (left) and left- and right-endpoints pointvalued linear models (right).

\section{Application to Real Data}

In this section, we use the interval-valued linear model to investigate the relationship between temperature and latitude. The data are the highest and the lowest temperatures of 15 European cities on 14 August, 2012, as shown in Table 3 and Figure 3.

Table 3. Temperatures $\left({ }^{\circ} \mathrm{C}\right)$ and latitudes $\left({ }^{\circ}\right)$ of 15 European cities on 14 of August, 2012.

\begin{tabular}{|c|c|c|c|}
\hline City & Latitude & Highest Temp. & Lowest Temp. \\
\hline Athens & 38 & 24 & 34 \\
\hline Madrid & 40.4 & 19 & 31 \\
\hline Istanbul & 41 & 23 & 30 \\
\hline Roma & 41.9 & 23 & 33 \\
\hline Marsaille & 43.3 & 19 & 31 \\
\hline Geneve & 46.25 & 13 & 28 \\
\hline Paris & 48.8 & 19 & 26 \\
\hline Brussel & 50.8 & 14 & 25 \\
\hline London & 51.5 & 14 & 21 \\
\hline Berlin & 52.5 & 13 & 23 \\
\hline Moscow & 55.75 & 14 & 24 \\
\hline Stockholm & 59.3 & 12 & 20 \\
\hline St. Petersburg & 59.9 & 13 & 22 \\
\hline Bergen & 60.4 & 14 & 20 \\
\hline Reykjavik & 64 & 11 & 17 \\
\hline
\end{tabular}

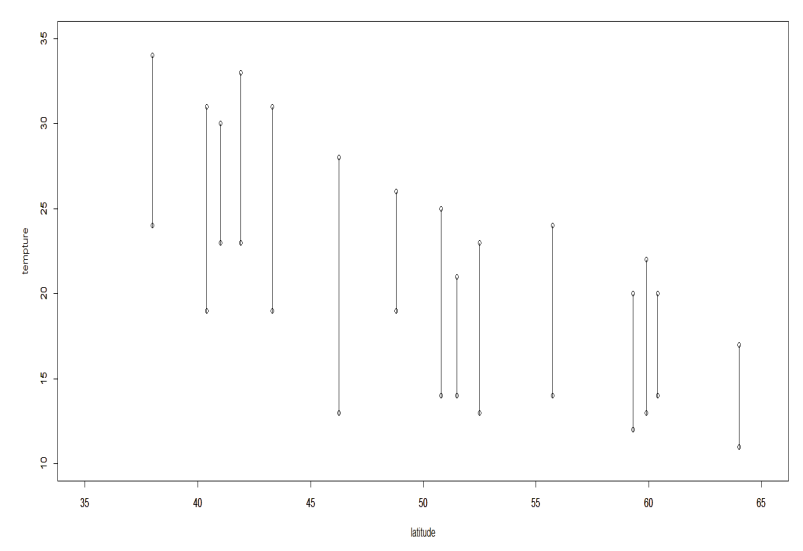

Fig. 3. Temperatures (in the form of interval) and latitudes of 15 European cities. Each line segment represents the temperature interval of each city. 
Suppose that the temperature $y$ (interval-valued) and the latitude $x$ (real-valued) can be represented by the interval-valued linear model (1), that is

$$
E\left(y_{i}\right)=\beta_{1}+x_{i} \beta_{2}, i=1,2, \cdots, 15 .
$$

The LSE of $\beta_{1}, \beta_{2}$ may be obtained via (3). The linear relationship (shown in Figure 4) between temperature $y$ and latitude $x$ is

$$
y=[39.03-0.45 x, 56.01-0.60 x] .
$$

Figure 4 indicates that as the latitude increases the temperature decreases, and the daily difference in temperature also tends to decrease.

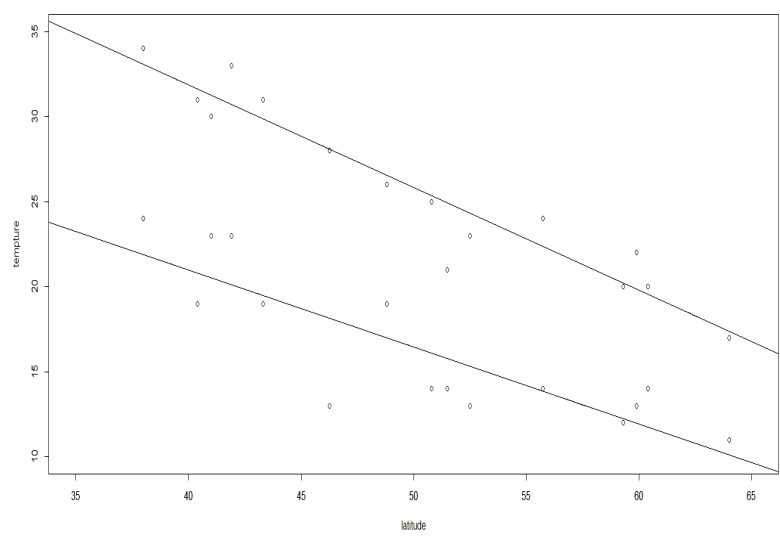

Fig. 4. Data and linear relationship of temperature and latitude of 15 cities in Europe on 14 August, 2012. The two lines represent the interval-valued linear regression function $y=[39.03-0.45 x, 56.01-0.60 x]$.

\section{Conclusions}

The linear regression model, which assumes a linear relationship between a random input variables and a few input variables, plays an important role in statistics. However, many phenomena are better described by an interval-valued random variable determined by a few real-valued random variables, e.g., temperature, stock price, service life of a kind of products. The relation between the interval-valued data and a few real-valued data can sometimes be expressed by a linear model. Therefore, we need a new type of statistical model to describe this kind of problems. In this paper, we introduced such a statistical model: the interval-valued linear model, which considers interval-valued observations determined by real-valued variables in a linear way.

Interval-valued random variables are a special kind of set-valued random variables taking values in the set of compact convex subsets of $\mathbb{R}^{1}$. In this paper, we investigated the theory in the general set-valued framework first, before focusing on the interval-valued random variables, in order to obtain some more general theoretical results. In particular, we recalled the definition of variance and covariance of set-valued random variables based on the $d_{p}$ metric for sets and the $D_{p}$ metric for set-valued random variables. We then introduced the interval-valued linear model and its LSE, proved the unbiasedness of the LSE and computed the covariance matrix of this estimator. We also showed that the best linear unbiased estimation does not exist in general, but the LSE is the unique best binary linear unbiased estimation (BBLUE). The performances of the estimation method were illustrated using simulation experiments, and compared to those of the simple approach that consists in fitting two separate linear models using the endpoints of output intervals. The obtained results suggest that our approach yields better forecasting performance. Finally, we gave an example of the interval-valued linear model explaining how temperature is related by latitude. This short example shows how our model can be used and what type of practical problem can be solved using the interval-valued linear model.

\section{References}

1. Z. Artstein and R. A. Vitale, "A strong law of large numbers for random compact sets", Annals of Probability, 3, 879-882 (1975).

2. J. P. Aubin and H. Franbowska, Set-Valued Analysis, Birkhauser (1990).

3. R. Aumann, "Integrals of set valued functions", Journal of Mathematical Analysis and Applications, 12, 112 (1965).

4. A. Blanco, N. Corral, G. Gonzalez-Redriguez and M. A. Lubiano, "Some properties of the $d_{K}$-variance for interval-valued sets", D. Dubois et al. (Eds.): Soft Methods for Handling Variability and Imprecision, 
ASC 48, 331-337 (2008).

5. A. Blanco-Fernandez, N. Corral and G. GonzalezRedriguez, "Estimation of a flexible simple linear model for interval data based on set arithmetic", Computational Statistics and Data Analysis, 55, 25682578 (2011).

6. A. Blanco-Fernandez, A. Colubi and G. GonzalezRedriguez, "Confidence sets in a linear regression model for interval data", Journal of Statistical Planning and Inference, 142, 1320-1329 (2012).

7. B. R. Clarke, Linear Model: the Theory and Application of Analysis of Variance, Wiley (2008).

8. T. Denoeux and M.-H. Masson, "Multidimensional scaling of interval-valued dissimilarity data", Pattern Recognition Letters, 21, 83-92 (2000).

9. Denoeux, T. and M.-H. Masson, "Principal component analysis of fuzzy data using autoassociative neural networks", IEEE Transactions on Fuzzy Systems, 12 (3), 336-349 (2004).

10. P. Diamond and P. Kloeden, Metric Space of Fuzzy Sets, World Scientific (1994).

11. F. Hiai and H. Umegaki, "Integrals, conditional expectations and martingales of multivalued functions", Journal of Multivariate Analysis, 7, 149-182 (1977).

12. A. Maia, F. Carvalho and T. B. Ludermir, "Forecasting models for interval-valued time series", Neurocomputing, 71, 3344-3352 (2008).

13. M. Masson and T. Denoeux, "Multidimensional scaling of fuzzy dissimilarity data", Fuzzy Sets and Systems, 128 (3): 339-352 (2002).

14. H. L. Hsu and B. Wu, "Evaluating forecasting performance for interval data", Computers and Mathematics with Applications, 56, 2155-2163 (2008).

15. T. L. Lai and H. Xing, Statistical Model and Methods for Financial Markets, Springer (2007).

16. S. Li, Y. Ogura, "Convergence of set valued sub- and super-martingales in the Kuratowski-Mosco sence", Annals of Probability, 26, 1384-1402 (1998).

17. S. Li and Y. Ogura, "Convergence of set valued and fuzzy valued martingales", Fuzzy Sets and System,
101, 453-461 (1999).

18. S. Li, Y. Ogura and V. Kreinovich, Limit Theorems and Applications of Set-Valuded and Fuzzy SetValued Random Variables, Kluwer Academic Publishers (Now Springer), Dordrecht (2002).

19. I. Molchanov, Theory of Random Sets, Springer (2005).

20. B. Sinova, A. Colubi, M. A. Gil and G. GonzalezRodriguez, "Interval arithmetic-based simple linear regression between interval data: Discussion and sensitivity analysis on the choice of the metric," Information Sciences, 199, 109-124 (2012).

21. H. Tanaka and H. Lee, "Interval regression analysis by quadratic programming approach", IEEE Transactions on Fuzzy Systems, 6 (4) (1998).

22. F. Tseng, G. Tzeng, H. Wu and B. Yuan, "Fuzzy ARIMA model for forecasting the foreign exchange market", Fuzzy Sets and Systems, 118, 9-19 (2001).

23. Vital, R.A., " $L_{p}$ metrics for compact, convex sets", Journal of Approximation Theory, 45 (3), 280-287 (1985).

24. X. Wang and S. Li, "The interval autoregressive time series model", Proceeding of IEEE-FUZZ International Conference, 2528-2533 (2011).

25. X. Wang and S. Li, "Stationary set-valued and interval-valued time series", preprint (2011).

26. X. Wang, S. Li and T. Denoeux, "Interval-valued linear model", Proceeding of 8th International Symposium on Imprecise Probability: Theories and Applications, Compiegne, France (2013).

27. X. Yang and $\mathrm{S}$. Li, "The $D_{p}$-metric space of set-valued random variables and its application to covariances", International Journal of Innovative Computing, Information and Control, 1, 73-82 (2005).

28. X. Yang, The $D_{p}$-metric space of set-valued random variables and its applications, Dissertation for Sciences Master's Degree (2005).

29. W. Zhang, S. Li, Z. Wang and Y. Gao, Set-Valued Stochastic Processes, Science Publisher (in Chinese) (2007). 